\title{
Ultrafast laser induced precessional dynamics in antiferromagnetically coupled ferromagnetic thin films
}

\author{
Jingyuan Zhou, Susmita Saha $\odot,{ }^{*}$ Zhaochu Luo $\odot$, Eugenie Kirk, Valerio Scagnoli $\odot$, and Laura J. Heyderman $\odot$ \\ Laboratory for Mesoscopic Systems, Department of Materials, ETH Zurich, 8093 Zurich, Switzerland \\ and Laboratory for Multiscale Materials Experiments, Paul Scherrer Institute, 5232 Villigen PSI, Switzerland
}

(Received 28 February 2020; revised manuscript received 5 May 2020; accepted 28 May 2020; published 22 June 2020)

\begin{abstract}
Antiferromagnetically coupled ferromagnetic thin films have recently attracted significant attention in magnonics because of the possibility to tune the spin-wave dispersion by altering the interlayer exchange coupling. To implement such coupled films in magnonic devices, a detailed understanding of the precessional dynamics of magnetization in such systems is required. Here, we present a systematic characterization of the precessional dynamics for systems with the layer magnetization going from nearly antiparallel to parallel alignment in a magnetic field. Experimentally, we have measured the ultrafast-laser-induced magnetization precession in samples with different interlayer exchange coupling strengths using the time-resolved magnetooptical Kerr effect. In our measurements, in addition to the acoustic and optical modes, an extra mode is observed that is due to the laser-induced decoupling of the two ferromagnetic layers. The observed precessional dynamics is in good agreement with our theoretical model based on the Landau-Lifshitz-Gilbert equation, and can be separated into three different field regions determined by the relationship between the Zeeman energy and the energy associated with the interlayer exchange coupling. This systematic paper, which gives a detailed description of how the interlayer exchange coupling and Zeeman energy influence the precessional dynamics, provides an important guide to implement antiferromagnetically coupled films in functional magnonic devices.
\end{abstract}

DOI: 10.1103/PhysRevB.101.214434

\section{INTRODUCTION}

Magnetic trilayer films, in which two ferromagnetic (FM) layers can couple antiferromagnetically via a nonmagnetic spacer layer, led to the discovery of the giant magnetoresistance effect $[1,2]$. Since then, these systems have attracted significant attention because of their potential applications such as magnetic-field sensors [3,4] and magnetic random access memory [5]. These trilayer films are also referred to as synthetic antiferromagnets [6], where the large tunability of the interlayer coupling in these systems has been important for many related research areas such as the motion of domain walls [7], solitons [8], skyrmions [9], and the use of synthetic antiferromagnets for flexible spintronics and biotechnology applications [10,11]. The tunability of trilayer films arises from the oscillatory nature of the Ruderman-KittelKasuya-Yosida interlayer exchange coupling (IEC) [12,13], which means that not only an antiferromagnetic coupling but also a ferromagnetic coupling can be achieved by tuning the thickness of the spacer layer [14-16]. In addition, with an antiferromagnetic IEC, the precessional dynamics of the magnetization involves more modes, which is more complex than in a single FM layer [17,18]. Accordingly, the spinwave dispersion relations in these systems are also modified $[19,20]$, which are important for their potential applications in magnonics. For example, the antiferromagnetic IEC has

*susmitasaha.phy@gmail.com been proposed to give a nonreciprocal spin-wave dispersion in the Damon-Eshbach geometry [21-23]. This nonreciprocity could be exploited to build nonreciprocal magnonic devices that function in a similar manner to isolators and circulators that are widely used in electronics or photonics [24-26].

Due to the presence of the IEC, the precessional dynamics of the trilayer films can be described as a coupled precession of the two FM layers. The coupled dynamics has two characteristic modes; the acoustic mode where the magnetization in the two layers precess in phase and the optical mode where the magnetization in the two layers precess $\pi$ out of phase [27]. Theoretically, one can describe the precessional dynamics using the formalism of statistical thermodynamics, as described by Liu et al. [17], or using the Landau-LifshitzGilbert (LLG) equation [28]. Experimentally, one can measure the frequencies of both the acoustic and optical modes in the frequency and time domains using broadband ferromagnetic resonance (FMR) $[17,18,27,29,30]$ and the timeresolved magneto-optical Kerr effect (TRMOKE) [31-34], respectively.

In this paper, we exploit the competition between the Zeeman energy and the energy associated with the IEC to provide a comprehensive understanding of different precessional modes in the trilayer films. To do so, we measure the precessional dynamics of three samples with different IEC strengths in the same range of applied magnetic field. We find that the observed precessional dynamics can be divided into three field regions, depending on the relationship between the Zeeman energy and the energy associated with the IEC. 
TABLE I. Layer thicknesses for the three samples $\operatorname{CoFeB}\left(t_{2}\right) / \mathrm{Ru}\left(t_{\mathrm{Ru}}\right) / \mathrm{CoFeB}\left(t_{1}\right) / \mathrm{Al}$.

\begin{tabular}{lccc}
\hline \hline Sample & $t_{1}(\mathrm{~nm})$ & $t_{2}(\mathrm{~nm})$ & $t_{\mathrm{Ru}}(\mathrm{nm})$ \\
\hline 1 & 5.0 & 2.5 & 1.0 \\
2 & 5.0 & 2.5 & 0.5 \\
3 & 5.0 & 5.0 & 0.4 \\
\hline \hline
\end{tabular}

To quantitatively verify the experimental results, we have calculated both the frequency and phase of the acoustic and optical modes for our systems using the LLG equation. We find that the experimental and theoretical results are in good agreement and, in addition to the acoustic and optical modes, we have observed another mode at relatively small applied magnetic fields with a high laser excitation fluence. This mode behaves in a similar manner to the FMR mode in a single FM film and is due to laser-induced decoupling between the two FM layers. The existence of this additional mode indicates that we have a further degree of freedom to control the precessional dynamics in antiferromagnetically coupled trilayer films. In particular, by changing the laser fluence, we can switch this additional mode on and off.

This paper is organized as follows: In Sec. II, we present experimental details of the sample fabrication, magnetization characterization, and TRMOKE measurements. In Sec. III, we introduce the theoretical formalism to calculate the frequency and phase of the precessional dynamics. We then present our experimental results in Sec. IV, where we find the three precessional modes. Finally, we summarize our results in Sec. V, where we interpret the different precessional dynamics in the three field regions in terms of the competition between the Zeeman energy and the energy associated with the IEC. In the Appendix, we present the detailed formalism used to solve the precessional dynamics.

\section{EXPERIMENTAL DETAILS}

\section{A. Sample manufacture and characterization}

Three different $\mathrm{CoFeB} / \mathrm{Ru} / \mathrm{CoFeB}$ trilayer films were deposited using DC magnetron sputtering at a base pressure of $\sim 2-3 \times 10^{-8}$ mbar. The layer thicknesses for the trilayer samples are listed in Table I. Samples 1 and 2 incorporate two FM layers with different thicknesses where $t_{1}=2 t_{2}$, which are referred to as asymmetric samples. Sample 3 includes two FM layers with the same thickness, which is referred to as the symmetric sample. Going from sample 1 to 3 , the thickness of the Ru layer, $t_{\mathrm{Ru}}$, is varied to give a different IEC strength for the three samples. All samples were coated with a 2-nm Al layer to prevent oxidization.

The magnetic properties of the samples were characterized at room temperature with an MPMS3 Superconducting Quantum Interference Device-Vibrating Sample Magnetometer. The in-plane hysteresis loops for all samples are shown in Fig. 1(a). Because of the antiferromagnetic IEC, the magnetizations of the two FM layers prefer to align antiparallel, which leads to a reduction of the net magnetization at zero field compared with the saturation magnetization. For samples 1 and 2, since the thicknesses of the two FM layers are different,
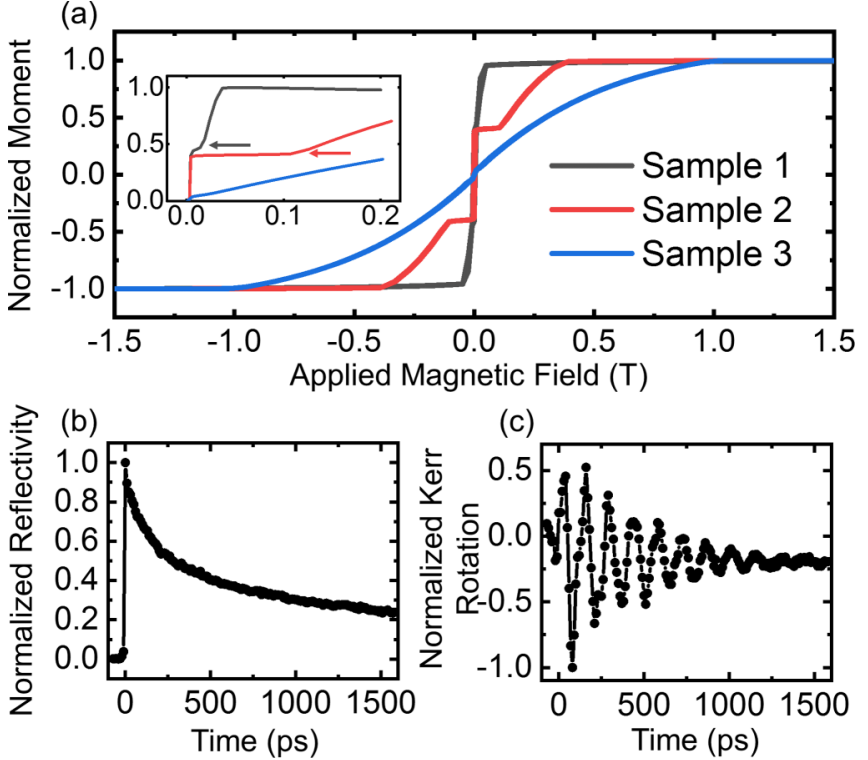

(c)

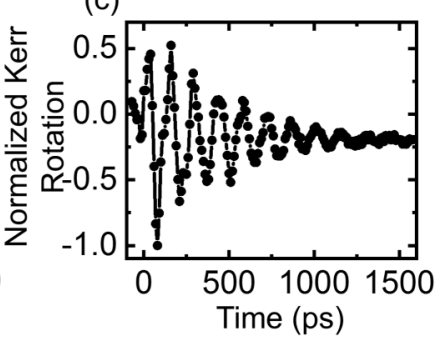

FIG. 1. (a) In-plane hysteresis loops of the three samples recorded in a magnetic field range of -1.5 to $+1.5 \mathrm{~T}$. Inset: Magnified view of the hysteresis loops between 0 and $0.2 \mathrm{~T}$. The two magnetization plateaus for samples 1 and 2 are indicated with arrows. Normalized time-resolved (b) reflectivity and (c) Kerr rotation data of sample 2 measured at a magnetic field of $150 \mathrm{mT}$.

there is a net magnetization close to zero magnetic field, which is observed as a magnetization plateau in Fig. 1(a) indicated with arrows in the inset. For sample 3, the two FM layers have the same thickness. Therefore, there is no net magnetization close to zero magnetic field. With increasing applied magnetic field, the magnetizations of the two FM layers start to align along the direction of the applied field, which results in the recovery of the net magnetization for all three samples.

\section{B. TRMOKE measurement}

The precessional dynamics of the samples was measured using TRMOKE based on an all-optical pump-probe technique. The laser light is produced with an optical fiber laser source, which has a central wavelength of $1030 \mathrm{~nm}$, pulse width of $\sim 150 \mathrm{fs}$, and repetition rate of $200 \mathrm{kHz}$. To perform the pump-probe experiment, the laser beam is divided into two paths. One is guided through a barium borate crystal to generate second-harmonic laser light $(515 \mathrm{~nm})$, which is used to excite the magnetization dynamics. The other beam is used to probe the magnetization dynamics. Both of the beams are focused onto the sample using a microscope objective with a numerical aperture of 0.65 . With different time delays between the pump and probe beams, the magnetization dynamics over time can be measured. The reflectivity and the out-of-plane component of the magnetization are measured using a balanced photodetector. The signals are retrieved using a lock-in amplifier, with an optical chopper modulating the pump beam close to $1000 \mathrm{~Hz}$. The external magnetic field is applied to the sample with a permanent magnet. For the induced precessional dynamics, we consider only the in-plane component of the external applied magnetic field, which we refer to as $\mathbf{H}^{\text {ext }}$. 


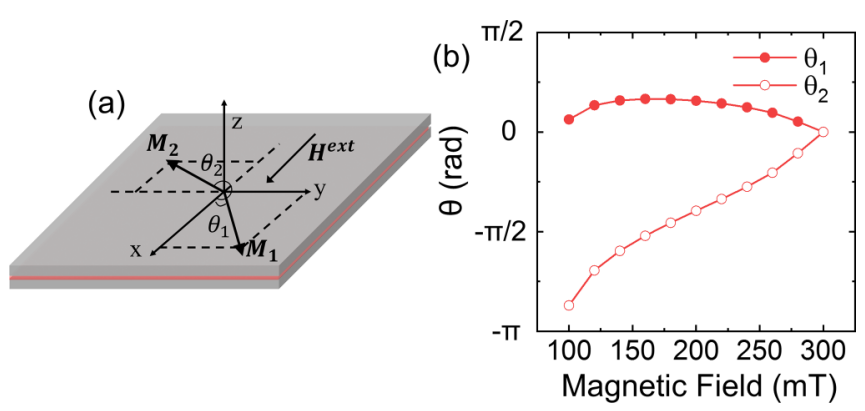

FIG. 2. (a) Schematic of the magnetization configuration of the trilayer film and the coordinate system. (b) Magnetization orientation in the two layers, $\theta_{1}$ and $\theta_{2}$, of sample 2 as a function of applied magnetic field.

Typical measurements of time-resolved reflectivity and Kerr rotation from sample 2 are shown in Figs. 1(b) and 1(c), respectively. From the reflectivity measurements, the time zero is determined, where the pump and probe pulses have no time delay with respect to each other. An exponential decay is observed in the reflectivity signal and, for the Kerr rotation signal, there is a damped sinusoidal oscillation, which corresponds to a damped precessional motion of the magnetization.

\section{THEORETICAL DESCRIPTION OF PRECESSIONAL DYNAMICS}

In this section, we present the theoretical description of the precessional dynamics in trilayer films. We first solve the static magnetization configuration. Then the obtained static magnetization is used to solve the LLG equation, which gives the frequency and phase of the precessional dynamics. In our model, we consider uniformly magnetized FM layers with the following energy terms:

$$
E=E^{\mathrm{IEC}}+\sum_{j=1,2}\left(E_{j}^{\mathrm{Z}}+E_{j}^{\mathrm{D}}\right),
$$

where $E^{\mathrm{IEC}}, E^{\mathrm{Z}}$ and $E^{\mathrm{D}}$ are the IEC, Zeeman, and demagnetization energies, respectively. Since the sputtered $\mathrm{CoFeB}$ layer is amorphous, we do not consider any crystalline anisotropy here. The detailed expressions for each of the terms are as follows:

$$
E=J \frac{\mathbf{M}_{1} \cdot \mathbf{M}_{2}}{\left|\mathbf{M}_{1}\right|\left|\mathbf{M}_{2}\right|}-\sum_{j=1,2} \mu_{0} t_{j}\left[\mathbf{H}^{\mathrm{ext}} \cdot \mathbf{M}_{j}+\frac{1}{2}\left(\mathbf{M}_{j} \cdot \hat{z}\right)^{2}\right] .
$$

According to the schematic of the magnetizations in the trilayer films shown in Fig. 2(a), $\mathbf{M}_{1}$ and $\mathbf{M}_{2}$ (or $\mathbf{M}_{j}$, where $j=1,2$ ) are the magnetizations of the top and bottom FM layers, both of which are in plane with the saturation value of $M . \mathbf{H}^{\text {ext }}$ is also in plane, with the absolute value of $H$. The antiferromagnetic IEC is quantified by the exchange constant $J$, with $J>0 . t_{j}$ is the thickness of each FM layer, $\mu_{0}$ is the vacuum permeability, and $\hat{z}$ is the unit vector along the $z$ axis. In this model, we neglect the dipole-dipole interaction between the two FM layers, since we consider the magnetization dynamics inside each FM layer to be uniform.

\section{A. Static magnetization configuration}

To determine the static magnetization configuration, the total energy given in Eq. (2) is minimized with respect to the orientation of $\mathbf{M}_{1}$ and $\mathbf{M}_{2}$. As illustrated in Fig. 2(a), the static magnetization configuration is described by angles $\theta_{1}$ and $\theta_{2}$, which are between the $x$ axis and $\mathbf{M}_{1}$ and $\mathbf{M}_{2}$, respectively. Here, $E^{D}$ vanishes, and only the energy terms related to $E^{\mathrm{IEC}}$ and $E^{\mathrm{Z}}$ need to be considered, as the magnetizations are in plane. For the symmetric sample $\left(t_{1}=t_{2}=t\right)$, the following analytic expression of the static magnetization configuration can be obtained, given that $\theta_{1}=-\theta_{2}=\theta$ :

$$
\cos \theta=\frac{\mu_{0} H M t}{2 J} .
$$

For the asymmetric sample $\left(t_{1}=2 t_{2}=2 t\right)$, only numerical values of $\theta_{1}$ and $\theta_{2}$ can be obtained. These are plotted against the magnetic field for sample 2 in Fig. 2(b), using the saturation magnetization $M=1042 \mathrm{kA} / \mathrm{m}$ and $J=0.5 \mathrm{~mJ} / \mathrm{m}^{2}$.

Two important pieces of information can be obtained from considering the static magnetization configuration. The first is the strength of the IEC, which can be estimated from the $H$ required to align both magnetizations parallel, which is the saturation field of the sample. From the hysteresis loops in Fig. 1(a), it can be deduced that $J_{1}<J_{2}<J_{3}$, where the subscripts correspond to the different samples. The second piece of information is that there is a significant difference between asymmetric and symmetric samples in terms of the static magnetization configuration. Since the Zeeman energy $E^{\mathrm{Z}}$ is proportional to the film thickness, $\left|\theta_{1}\right| \neq\left|\theta_{2}\right|$ for the asymmetric sample, whereas $\left|\theta_{1}\right|=\left|\theta_{2}\right|$ for the symmetric sample.

\section{B. Precessional dynamics}

To determine the precessional dynamics of the trilayer film, we consider the differential equations involving the two FM layers based on the LLG equation,

$$
\frac{d \mathbf{M}_{j}}{d t}=-\gamma \mu_{0} \mathbf{M}_{j} \times \mathbf{H}_{j}^{\mathrm{eff}},
$$

where $\mathbf{H}_{j}^{\text {eff }}$ is the total effective magnetic field that drives the magnetization precession and $\gamma$ is the gyromagnetic ratio. The effective field in the two FM layers can then be obtained from [35]

$$
\mathbf{H}_{j}^{\mathrm{eff}}=-\frac{1}{\mu_{0} t_{j}} \frac{\partial E}{\partial \mathbf{M}_{j}} .
$$

To solve Eq. (4), the following ansatz is used [21]:

$$
\mathbf{M}_{j}(t)=\mathbf{M}_{j}^{\mathrm{S}}+\mathbf{m}_{j} e^{i \omega t} .
$$

Here, $\mathbf{M}_{j}^{\mathrm{S}}$ is the static magnetization, which is obtained by minimizing Eq. (2), $\mathbf{m}_{j} e^{i \omega t}$ is the dynamic magnetization, and $\omega$ is the precession frequency. For convenience, we give the final expression of the precession frequency $f$ in $\mathrm{Hz}$, with $f=$ $\omega / 2 \pi$, and the detailed calculations are given in the Appendix.

For the symmetric sample, the precession frequency and the phase of the dynamic $m_{j z}$ components for the acoustic and 
optical modes are

$$
\begin{aligned}
f_{\mathrm{a}}= & \frac{\gamma \mu_{0}}{2 \pi} \sqrt{H(H+M \cos \theta)}, \\
\left(m_{1 z}, m_{2 z}\right)= & k_{1} e^{i \omega_{\mathrm{a}} t}(1,1), \\
f_{\mathrm{o}}= & \frac{\gamma \mu_{0}}{2 \pi}\left[H^{2}+2 A^{2} M^{2}+H M \cos \theta-4 A H M \cos \theta\right. \\
& \left.-2 A M^{2} \cos (2 \theta)+2 A^{2} M^{2} \cos (2 \theta)\right]^{\frac{1}{2}}, \\
\left(m_{1 z}, m_{2 z}\right)= & k_{2} e^{i \omega_{0} t}(-1,1),
\end{aligned}
$$

with $A=J /\left(\mu_{0} t M^{2}\right)$. Here $f_{\mathrm{a}}$ and $f_{\mathrm{o}}$ are the frequencies for the acoustic and optical modes, and $k_{1}$ and $k_{2}$ are related to the amplitude of precession. From the expressions of $m_{j z}$, it can be seen that $m_{1 z}$ and $m_{2 z}$ are in phase for the acoustic mode, whereas they have a $\pi$-phase shift for the optical mode.

For the asymmetric samples with $t_{1}=2 t$ and $t_{2}=t$, a general analytic solution of the precessional dynamics cannot be obtained (details given in the Appendix). It is only in the saturated state, with $\mathbf{M}_{1}$ and $\mathbf{M}_{2}$ aligned with the applied magnetic field, that there are analytic expressions for both the precession frequency and phase as follows:

$$
\begin{aligned}
f_{\mathrm{a}} & =\frac{\gamma \mu_{0}}{2 \pi} \sqrt{H(H+M)}, \\
\left(m_{1 z}, m_{2 z}\right) & =k_{1} e^{i \omega_{\mathrm{a}} t}(1,1), \\
f_{\mathrm{o}} & =\frac{\gamma \mu_{0}}{2 \pi} \sqrt{(H-3 A M / 2)(H+M-3 A M / 2)}, \\
\left(m_{1 z}, m_{2 z}\right) & =k_{2} e^{i \omega_{0} t}\left(-\frac{1}{2}, 1\right) .
\end{aligned}
$$

In the unsaturated state, only numerical values of the precession frequency can be obtained, which will be presented in Sec. IV. Expressions for $m_{j z}$ cannot be determined in this case.

\section{RESULTS AND DISCUSSION}

\section{A. Precessional dynamics of the samples}

The precessional dynamics of the samples is measured using TRMOKE on varying the applied magnetic field $\left(\mu_{0} H\right)$ from 100 to $300 \mathrm{mT}$. Representative time domain data of the three samples for three different applied magnetic fields of 110, 160, and $290 \mathrm{mT}$ are shown in Figs. 3(a)-3(c). The ultrafast laser pulse first induces an ultrafast demagnetization, which is followed by a fast remagnetization process [36,37]. After these two processes, the magnetization starts to precess around the equilibrium magnetic field [38]. For the case of multilayer films, the initial excitation caused by the laser pulse can be different for different layers, resulting in a nonuniform excitation [39]. For our experiments, since the time domain data is taken following the ultrafast demagnetization and remagnetization, which is several tens of picosecond after the excitation, this nonuniform excitation for the multilayers does not need to be considered when describing the precessional dynamics. The fast Fourier transform for each set of time domain data provides the frequency spectra shown in Figs. 3(d)3(f). Each peak in the frequency spectra corresponds to a specific mode of the precessional dynamics.

From both the time domain data and frequency spectra in Fig. 3, it can be seen that the precessional dynamics is
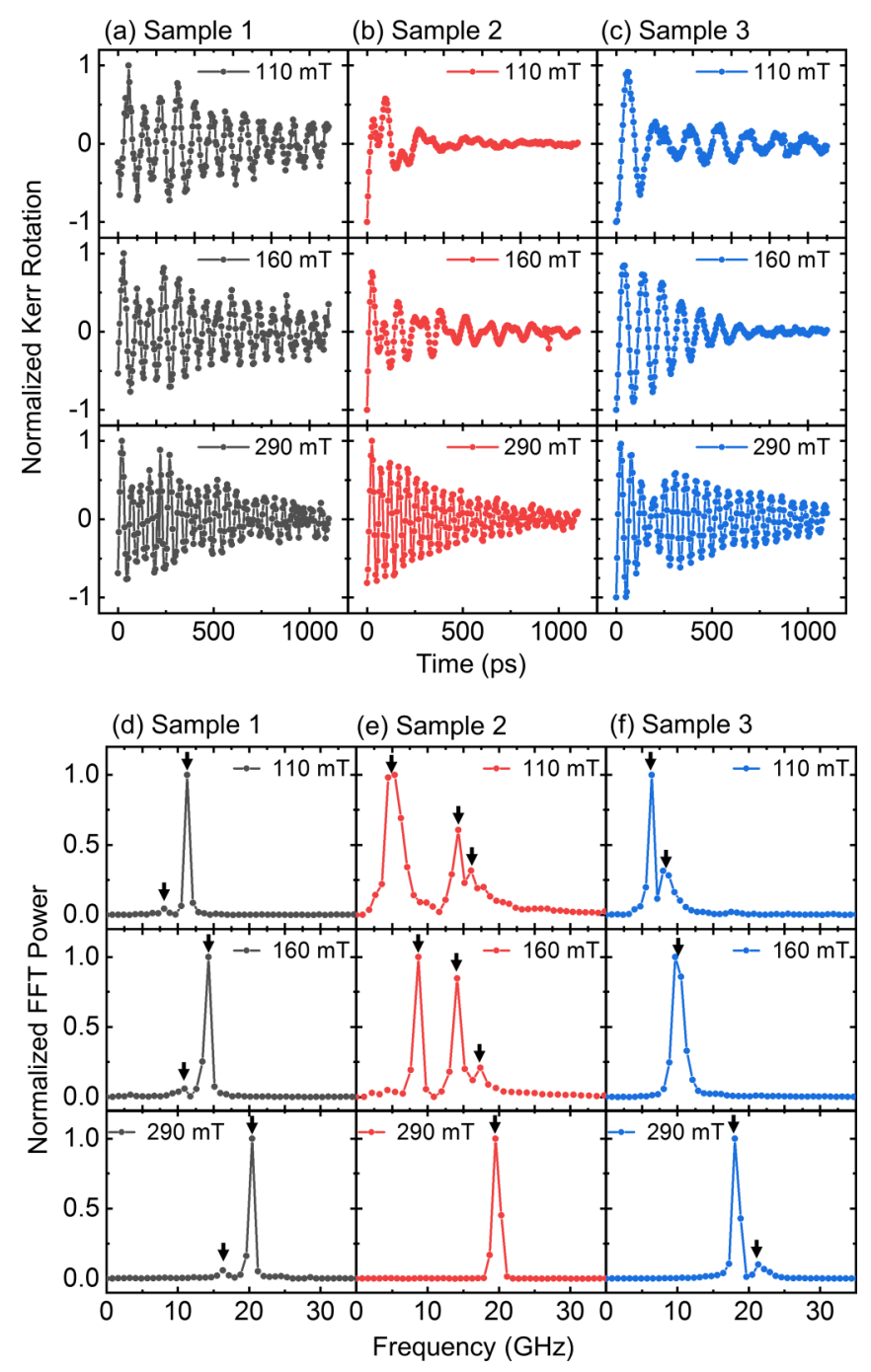

FIG. 3. Precessional dynamics induced by ultrafast laser excitation measured with TRMOKE for three samples at different applied magnetic fields. Samples 1 and 2 were measured at a laser excitation fluence of $10.0 \mathrm{~mJ} / \mathrm{cm}^{2}$, and sample 3 was measured at a laser excitation fluence of $13.4 \mathrm{~mJ} / \mathrm{cm}^{2}$. (a)-(c) Time-resolved Kerr rotation and (d)-(f) the corresponding frequency spectra, with each mode indicated with an arrow. Lines connecting the points are guides to the eye.

significantly different for the three different samples. For each sample, the precessional dynamics is also modified by varying the applied magnetic field. For sample 1, two modes are present at all applied fields. For sample 2, only one mode is present at $\mu_{0} H=290 \mathrm{mT}$, whereas three modes appear when the field is decreased to $160 \mathrm{mT}$ and continue to be present as the magnetic field is further reduced to $110 \mathrm{mT}$. For sample 3 , two modes are observed at 110 and $290 \mathrm{mT}$, whereas a single mode is observed at an intermediate field of $\mu_{0} H=160 \mathrm{mT}$.

To determine the origin of each mode, it is important to consider the frequency dependence of the different modes as a function of applied magnetic field for each sample [Figs. 4(a)4(c)]. In addition to the measurements, the frequency dependence of the characteristic modes of each sample is calculated [Figs. 4(d)-4(f)] using the equations given in Sec. III 


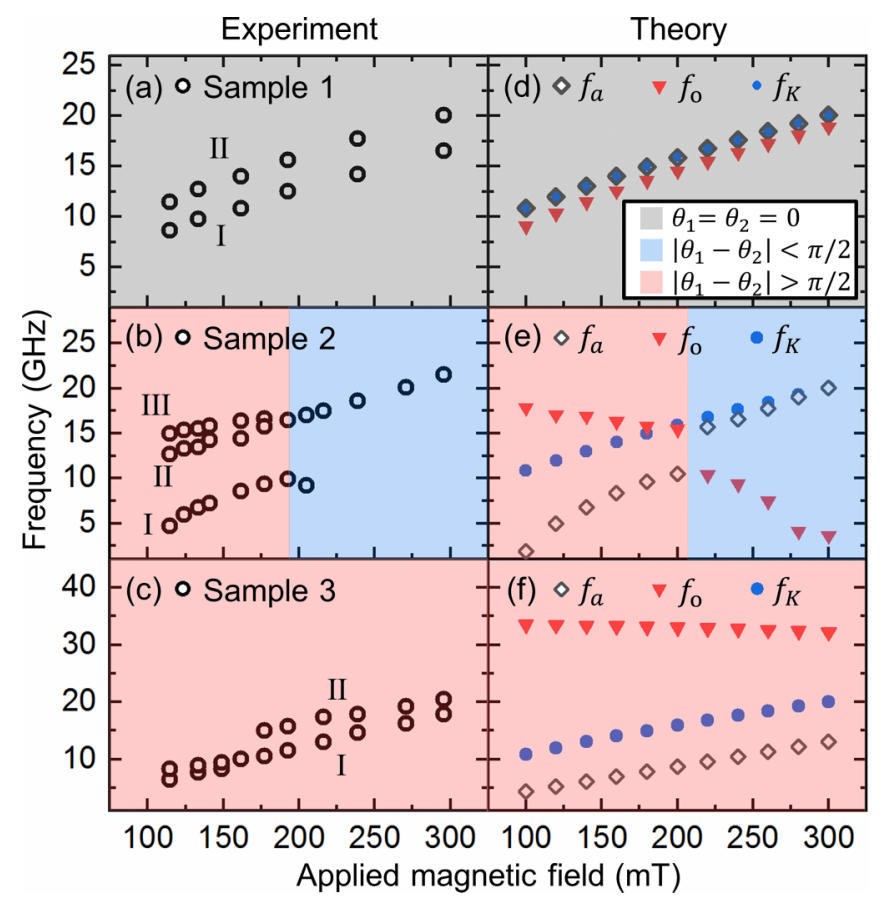

FIG. 4. Dependence of the frequency on the applied magnetic field for the different modes. (a)-(c) Experimental data: The different precessional modes are indicated with Roman numbers. (d)-(f) Theoretical data: $f_{\mathrm{a}}, f_{\mathrm{o}}$, and $f_{\mathrm{K}}$ are the frequencies of the acoustic mode, optical mode, and the FMR mode for a $7.5 \mathrm{~nm} \mathrm{CoFeB}$ film. The three different background colors indicate the three field regions given in the inset of (d). The error for each data point is on the order of 1-2 GHz, as shown in the Supplemental Material [40].

and the Appendix, with $M=1042 \mathrm{kA} / \mathrm{m}$ and $\gamma=1.76 \times$ $10^{11} \mathrm{rad} /(\mathrm{s} \cdot \mathrm{T})$. The IEC constant $J$ is estimated to be 0.1 , 0.5 and $2.7 \mathrm{~mJ} / \mathrm{m}^{2}$ for samples 1,2 , and 3 , respectively. We also determine a reference frequency $f_{\mathrm{K}}=\frac{\gamma \mu_{0}}{2 \pi} \sqrt{H(H+M)}$, which is the frequency of the FMR mode of a single CoFeB film of $7.5 \mathrm{~nm}$. By comparing the experimental and theoretical data, the origin of each mode can be identified.

For sample 1, as shown in Fig. 4(a), modes I and II are present for magnetic fields in the range of 100 to $300 \mathrm{mT}$, and the frequencies of both the modes increase with increasing the applied magnetic field, which is in agreement with our calculations for this sample [Fig. 4(d)]. By comparing the experimental and theoretical results in Figs. 4(a) and 4(d), we can therefore associate mode I with the optical mode, and mode II with the acoustic mode.

For sample 2, the observed and calculated frequency dependence is not linear, and two field regions can be identified that are separated by a discontinuity in the frequency dependence of the characteristic modes. Experimentally, a different number of modes are present in the two field regions. First, we discuss the field region with small magnetic field, where three modes are observed, and the calculations and experiments are in a qualitative good agreement, as shown in the red shaded area in Figs. 4(b) and 4(e). Through comparison, mode I can be identified as the acoustic mode and mode III as the optical mode. Mode II, which has a frequency similar to the FMR mode in a single layer $\mathrm{CoFeB}$ film, is neither an acoustic nor an optical mode, and we refer it to as a transient mode. As explained in Sec. IV B, the transient mode is due to the laser-induced decoupling between the two FM layers. We can now turn our attention to the higher magnetic field region of 190-300 mT. While the theoretical data [Fig. 4(e), blue region], in addition to the FMR mode $f_{K}$, exhibits two modes, experimentally the three modes are first reduced to two modes, and eventually only a single mode is observed with further increase of the field. Around $190 \mathrm{mT}$, there are discontinuities in the frequencies of the acoustic and optical modes, with mode I (III) changing to the optical (acoustic) mode.

Finally, for sample 3 the theoretical calculations [Fig. 4(f)] exhibit almost linear dependence of all the modes as a function of the applied magnetic field. However, the experimental data looks, at first glance, quite different. To reconcile our measurements with the calculations, first we note that the net MOKE signal of the optical mode $\left(m_{1 z}=-m_{2 z}\right)$ from the two equally thick FM layers is zero and therefore cannot be observed experimentally. Therefore, for $\mu_{0} H>175 \mathrm{mT}$, mode I, having a lower frequency than $f_{\mathrm{K}}$, is the acoustic mode and mode II is the transient mode. At magnetic fields $\mu_{0} H<175 \mathrm{mT}$, two modes are also observed, but with a smaller frequency difference. As their frequencies are smaller than $f_{\mathrm{K}}$, they cannot be attributed to the transient mode and they might be the result of the splitting of the acoustic mode due to domain formation, as reported for a similar system [41].

To summarize, the three samples exhibit significantly different precessional dynamics, which are in good agreement with the theoretical model except for the so-called transient mode. To elucidate its origin, we have performed fluencedependent TRMOKE measurements on samples 2 and 3, illustrated in the next section, which reveals how the transient mode evolves with varying the laser excitation fluence. Finally, in Sec. III C, we explain the relationship between the frequencies of the different modes in the three field regions [Fig. 4], i.e., which mode has a higher frequency, considering the competition between $E^{\mathrm{Z}}$ and $E^{\mathrm{IEC}}$.

\section{B. Origin of transient mode}

In this section, we present further measurements on samples 2 and 3 with varying laser fluence to support our claims that the presence of the transient mode is due to laser-induced decoupling of the FM layers due the suppression of the IEC.

The first hint for the presence of a laser-induced IEC suppression is the existence of a threshold in the laser fluence, which is not expected for characteristic modes of the trilayers. Indeed, as illustrated in Fig. 5, in both the samples, the transient mode (mode II) only appears above a laser fluence threshold of $8.2 \mathrm{~mJ} / \mathrm{cm}^{2}$ for sample 2 , and $13.4 \mathrm{~mJ} / \mathrm{cm}^{2}$ for sample 3 . The intensity of this mode increases further with the increase of the laser fluence (see the Supplemental Material [42] for more measurements). If we consider the energy deposited by the laser excitation, the first effect would be an increase in the temperature of the sample, which leads to a reduction of the IEC strength that is known to be dependent on temperature $[18,30]$. As a result, above a certain threshold level, the two FM layers become decoupled. In this case, the magnetization precession is only driven by the applied and the demagnetization fields. Hence, the frequency of the 
(a)
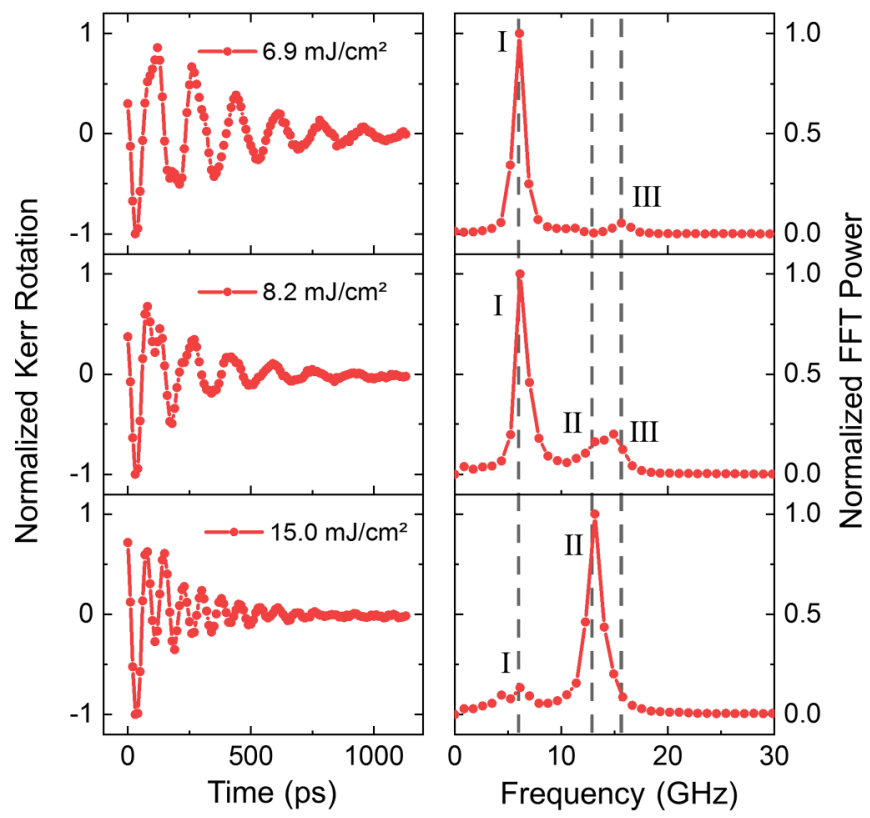

(b)

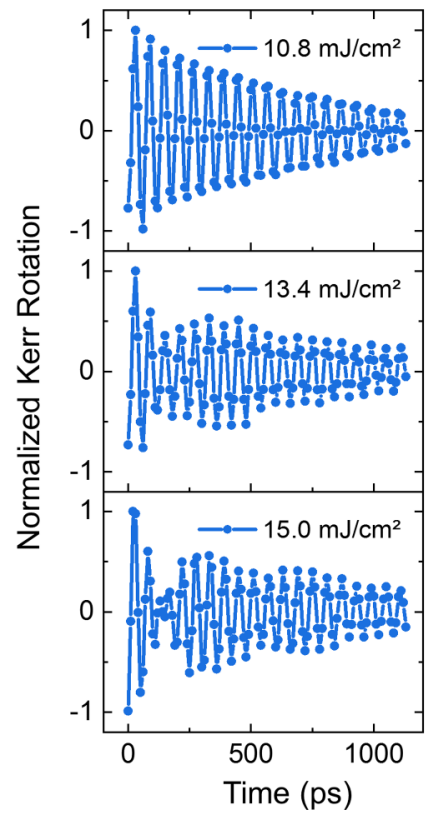

the Supplemental Material [43]). For sample 2, at the fluence of $8.2 \mathrm{~mJ} / \mathrm{cm}^{2}$, the time constant for the acoustic, optical, and transient modes are 253, 150, and 104 ps, respectively. For sample 3, at the fluence of $13.4 \mathrm{~mJ} / \mathrm{cm}^{2}$, the time constant for the acoustic and transient mode are 936 and 317 ps. In both cases, the time constant of the transient mode is shorter than the other characteristic modes, which is due to the recovery of the IEC when the heat dissipates away. A further confirmation of the extrinsic nature of the transient mode is that a higher fluence is required to excite the transient mode in sample 3 compared with sample 2 . This is because the IEC in sample 3 is stronger than sample 2 , and more energy must be deposited into the sample to decouple the two FM layers.

\section{Dependence of the precessional dynamics on the Zeeman energy and IEC}

In Sec. IV B, we clarified that the three modes observed are the acoustic, optical, and transient modes. We now explain in detail the relationship between the frequencies of the different modes as a function of the applied magnetic field.

We first determine how the IEC modifies the frequencies of the acoustic and optical modes, compared with the reference frequency $f_{\mathrm{K}}$. For the acoustic mode, its frequency $f_{\mathrm{a}}$ is modified by the IEC only because the IEC affects the static magnetization configuration, which is given by the $M \cos \theta$ term in Eqs. (7). In fact, this implies that any features present in the hysteresis loops will affect the precessional dynamics. In contrast, the frequency of the optical mode $f_{\mathrm{o}}$ depends on both the effective field from the IEC $\mathbf{H}^{\mathrm{IEC}}$, given several terms in Eqs. (7b) contain the constant $A$ that is related to the IEC, and the static magnetization configuration. Here it should be noted that, although Eqs. (7) are the analytic solution for sample 3, it can still provide some qualitative information for samples 1 and 2.

Knowing how the IEC modifies the frequency of acoustic and optical modes, we can now analyze the frequency dependence of different modes as a function of the applied magnetic field. In particular, we can explain the relationship between the frequencies of different modes. For this purpose, we divide the experimental data into three field regions that differ in the relative contributions from the Zeeman energy and IEC, and are indicated by different background colors in Fig. 4. The first field region (in gray) is for a saturated sample, where the Zeeman energy dominates. The second field region (in blue) is for a field below the saturation field but where the field is strong enough to have an almost parallel alignment of the layer magnetizations, with $\left|\theta_{1}-\theta_{2}\right|<\pi / 2$. The third field region (in red) is where the field is no longer strong enough to overcome the antiparallel alignment, with $\left|\theta_{1}-\theta_{2}\right|>\pi / 2$.

We begin with the precessional dynamics in the first field region [Fig. 4(a), gray region]. As can be seen for sample 1, in the first field region where the Zeeman energy dominates, the frequency relationship between different modes is $f_{\mathrm{o}}<$ $f_{\mathrm{a}}=f_{\mathrm{K}}$. For the acoustic mode, as previously stated, the IEC can only modify $f_{\mathrm{a}}$ if the static magnetization configuration is different from the saturated state. Since in this field region the sample is saturated, the acoustic mode has the same frequency as the FMR mode of a single layer CoFeB film. For the optical mode, the additional contribution from the effective field of

where $A_{i}, \tau_{i}, f_{i}$, and $t_{i}$ are the amplitude, time constant, frequency, and initial time for the corresponding mode (see 
the IEC $\mathbf{H}^{\text {IEC }}$ needs to be considered. For a saturated sample, $\mathbf{H}^{\mathrm{IEC}}$ [corresponding to the terms including $A$ in Eqs. (8b)] counteracts $\mathbf{H}^{\text {ext }}$, so $f_{\mathrm{o}}<f_{\mathrm{K}}$. This can be explained by the phase difference between the precessing magnetizations in the two layers in the optical mode. For the optical mode, an antiparallel alignment of the dynamic $m_{z}$ components in the two layers is favored by the antiferromagnetic IEC. In contrast, for the acoustic mode, the parallel alignment of the two dynamic $m_{z}$ components is not favored by the IEC. Therefore, less energy is required to excite the optical mode and, hence, the optical mode has a lower precession frequency than the acoustic mode. It should be mentioned that this argument is only valid for saturated samples, since the $\pi$-phase shift for the dynamic components is the only difference between the acoustic and optical modes.

Next, we explain the frequency relationship between different modes in the second field region [Fig. 4(b), blue region]. This field region extends for sample 2 from 200 to $300 \mathrm{mT}$. In this field region, the alignment of the magnetizations is not completely parallel because the Zeeman energy starts to exceed the energy associated with the IEC. Since the effect of $\mathbf{H}^{\text {ext }}$ counteracts $\mathbf{H}^{\mathrm{IEC}}$ for the optical mode [Eqs. (7b)], in this field region the frequency of the optical mode should decrease with increasing the applied magnetic field. At the same time, since the the two layer magnetizations are close to parallel alignment, the frequency of the acoustic mode is almost the same as $f_{\mathrm{K}}$. These trends in the optical and acoustic modes are reflected by the theoretical results in Fig. 4(e). As for the experimental data [Fig. 4(b)], the acoustic mode is in good agreement with the calculations, although the optical mode is only observed around $200 \mathrm{mT}$. We speculate that this is because the decrease of the frequency of the optical mode towards zero results in a gradual decrease of the intensity of this mode. As the optical mode already has quite a low intensity, it was therefore not possible to detect it on further increase of the applied magnetic field.

Finally, we explain the phenomena behind the dynamics in the third field region [Figs. 4(b) and 4(c), red regions]. This field region extends for sample 3 from 175 to $300 \mathrm{mT}$, and for sample 2 from 100 to $200 \mathrm{mT}$. In this field region, the static magnetization configuration is close to an antiparallel alignment of the layer magnetizations, because the energy associated with the IEC is greater than the Zeeman energy. For the acoustic mode, the nonparallel alignment between the two layer magnetizations makes $f_{\mathrm{a}}$ smaller than $f_{\mathrm{K}}$ [Eqs. (7)]. For the optical mode, since the energy associated with IEC is stronger than the Zeeman energy, the effective field from the IEC makes the frequency of the optical mode higher than $f_{\mathrm{K}}$. As a result, these give the frequency relationship $f_{\mathrm{a}}<f_{\mathrm{K}}$ for sample 3 and $f_{\mathrm{a}}<f_{\mathrm{K}}<f_{\mathrm{o}}$ for sample 2. For the small quantitative mismatch between the experimental and theoretical data, there could be various causes, such as a nonuniformity in the $\mathrm{Ru}$ layer.

\section{CONCLUSION}

We have used TRMOKE to characterize the ultrafastlaser-induced precessional dynamics in antiferromagnetically coupled trilayer films with different IEC strength. The precessional dynamics measured by TRMOKE depends on the relative thicknesses of the two FM layers. In asymmetric samples $\left(t_{1}=2 t_{2}\right)$, both the acoustic and optical modes are observed, whereas in the symmetric sample $\left(t_{1}=t_{2}\right)$ only the acoustic mode is observed. In addition to the two characteristic modes, a transient mode is also observed, which is due to the laser-induced decoupling of the two FM layers. Accordingly, the transient mode has the same precession frequency as the FMR mode in a single FM film, and is only present above a certain threshold of the laser excitation fluence.

We interpret the precessional dynamics in terms of a competition between the energy associated with the IEC and the Zeeman energy, and divide the precessional dynamics into three field regions depending on which energy term dominates. The first field region is for a saturated sample where the Zeeman energy dominates, and the frequency relationship between the modes is $f_{\mathrm{o}}<f_{\mathrm{a}}=f_{\mathrm{K}}$. The second field region is below the saturation field of the sample with $\left|\theta_{1}-\theta_{2}\right|<$ $\pi / 2$, and the relationship between the frequencies of different modes is $f_{\mathrm{o}}<f_{\mathrm{a}} \approx f_{\mathrm{K}}$. The third field region is again below the saturation field of the sample but with $\left|\theta_{1}-\theta_{2}\right|>\pi / 2$, and the frequency relationship is $f_{\mathrm{a}}<f_{\mathrm{K}}<f_{\mathrm{o}}$.

With this detailed understanding of how the IEC and Zeeman energy affect the precessional dynamics, we provide a foundation for further investigations of propagating spin waves in such systems and for using them to implement functional magnonic devices.

The data that support this study are available via the Zenodo repository [44].

\section{ACKNOWLEDGMENTS}

We thank Dr. S. Wintz and Dr. P. Derlet for fruitful discussions. This project is funded by the Swiss National Science Foundation (Project No. 200020_172774). S.S. acknowledges an ETH Zurich postdoctoral fellowship and Marie Curie actions for People COFUND program.

\section{APPENDIX: CALCULATION OF PRECESSIONAL DYNAMICS}

We present here the detailed formalism to determine the precessional dynamics based on the coupled LLG equations. We solve the dynamics for the asymmetric sample with $t_{1}=$ $2 t_{2}=2 t$, where the solutions are presented in Sec. III.

First, the precession part of the LLG equation [Eq. (4)] is rearranged to give

$$
\frac{d \mathbf{M}_{j}}{d t}+\gamma \mu_{0} \mathbf{M}_{j} \times \mathbf{H}_{j}^{\text {eff }}=0 .
$$

To solve this, we need to find the expressions for $\mathbf{M}_{j}$ and $\mathbf{H}_{j}^{\text {eff }}$. The three vector components of $\mathbf{M}_{j}$ in terms of $\theta_{j}$ are expressed as follows:

$$
\begin{aligned}
\mathbf{M}_{j}(t) & =\mathbf{M}_{j}^{\mathrm{S}}+\mathbf{m}_{j}(t) \\
& =\left(M \cos \theta_{j}, M \sin \theta_{j}, 0\right)+e^{i \omega t}\left(m_{j x}, m_{j y}, m_{j z}\right) .
\end{aligned}
$$

Here, the magnetization is divided into the static and dynamic parts, with the dynamic part having a time dependence of $e^{i \omega t}$. 
For the total effective field, we consider the contribution from the applied magnetic field, the demagnetization field, and the effective field from the IEC as follows:

$$
\begin{aligned}
\mathbf{H}_{j}^{\mathrm{eff}} & =\mathbf{H}_{j}^{\mathrm{Z}}+\mathbf{H}_{j}^{\mathrm{D}}+\mathbf{H}_{j}^{\mathrm{IEC}}, \text { where } \\
\mathbf{H}_{j}^{\mathrm{Z}} & =(H, 0,0), \\
\mathbf{H}_{j}^{\mathrm{D}} & =\left(0,0,-m_{j z}\right), \\
\mathbf{H}_{1}^{\mathrm{IEC}} & =\left(-\frac{A}{2} m_{2 x}-\frac{A}{2} M \cos \theta_{2},-\frac{A}{2} m_{2 y}-\frac{A}{2} M \sin \theta_{2},-\frac{A}{2} m_{2 z}\right), \\
\mathbf{H}_{2}^{\mathrm{IEC}} & =\left(-A m_{1 x}-A M \cos \theta_{1},-A m_{1 y}-A M \sin \theta_{1},-A m_{1 z}\right),
\end{aligned}
$$

Here, $A=J /\left(\mu_{0} t M^{2}\right)$ and $\mathbf{H}_{j}^{\mathrm{IEC}}$ corresponds to the effective field of the IEC for two FM layers of different thicknesses.

By linearizing Eq. (A1) with the expressions from Eqs. (A2) and (A3), we obtain a system of linear equations with the following form:

$$
\begin{aligned}
\gamma \mu_{0} L \mathbf{m}^{\mathrm{T}} & =\mathbf{c}, \\
\mathbf{m} & =\left(m_{1 x}, m_{1 y}, m_{1 z}, m_{2 x}, m_{2 y}, m_{2 z}\right),
\end{aligned}
$$

where $\mathbf{c}$ is a constant vector and $L$ is a $6 \times 6$ matrix expressed as follows:

$$
\left[\begin{array}{cccccc}
i \omega / \gamma \mu_{0} & 0 & -M \sin \theta_{1}+\frac{A}{2} M \sin \theta_{2} & 0 & 0 & -\frac{A}{2} M \sin \theta_{1} \\
0 & i \omega / \gamma \mu_{0} & H+M \cos \theta_{1}-\frac{A}{2} M \cos \theta_{2} & 0 & 0 & \frac{A}{2} M \cos \theta_{1} \\
-\frac{A}{2} M \sin \theta_{2} & -H+\frac{A}{2} M \cos \theta_{2} & i \omega / \gamma \mu_{0} & \frac{A}{2} M \sin \theta_{1} & -\frac{A}{2} M \cos \theta_{1} & 0 \\
0 & 0 & -A M \sin \theta_{2} & i \omega / \gamma \mu_{0} & A M \sin \theta_{1}-M \sin \theta_{2} & 0 \\
0 & 0 & A M \cos \theta_{2} & 0 & i \omega / \gamma \mu_{0} & H-A M \cos \theta_{1}+M \cos \theta_{2} \\
A M \sin \theta_{2} & -A M \cos \theta_{2} & 0 & -A M \sin \theta_{1} & -H+A M \cos \theta_{1} & i \omega / \gamma \mu_{0}
\end{array}\right] .
$$

For Eq. (A4), to have a nonzero solution of $\mathbf{m}$, we set the determinant of $L$ to zero and solve for $\omega$. For the case where $\omega$ can be solved analytically, solutions of $\omega$ are inserted back into matrix $L$ to obtain the solution of $\mathbf{m}$, given by Eqs. (8). From the expression of $\mathbf{m}$, the phase information of the two $m_{z}$ components can be obtained. For the case where $\omega$ has to be solved numerically, the general solution for $\mathbf{m}$ cannot be obtained. This is because any numerical values of $\omega$ are not the exact solutions for $\operatorname{det}|L|=0$. Therefore, if the numerical values of $\omega$ are inserted back into the matrix, $\operatorname{det}|L|$ will become zero, which cannot provide a nonzero solution of $\mathbf{m}$.

[1] M. N. Baibich, J. M. Broto, A. Fert, F. Nguyen Van Dau, F. Petroff, P. Etienne, G. Creuzet, A. Friederich, and J. Chazelas, Phys. Rev. Lett. 61, 2472 (1988).

[2] P. Grünberg, R. Schreiber, Y. Pang, M. B. Brodsky, and H. Sowers, Phys. Rev. Lett. 57, 2442 (1986).

[3] J. Daughton, J. Brown, E. Chen, R. Beech, A. Pohm, and W. Kude, IEEE Trans. Magn. 30, 4608 (1994).

[4] J. Daughton, J. Magn. Magn. Mater. 192, 334 (1999).

[5] S. Tehrani, J. Slaughter, E. Chen, M. Durlam, J. Shi, and M. DeHerren, IEEE Trans. Magn. 35, 2814 (1999).

[6] R. A. Duine, K.-J. Lee, S. S. P. Parkin, and M. D. Stiles, Nat. Phys. 14, 217 (2018).

[7] H. Saarikoski, H. Kohno, C. H. Marrows, and G. Tatara, Phys. Rev. B 90, 094411 (2014).

[8] R. Lavrijsen, J.-H. Lee, A. Fernández-Pacheco, D. C. M. C. Petit, R. Mansell, and R. P. Cowburn, Nature 493, 647 (2013).

[9] T. Dohi, S. DuttaGupta, S. Fukami, and H. Ohno, Nat. Commun. 10, 5153 (2019).

[10] T. Vemulkar, R. Mansell, A. Fernández-Pacheco, and R. Cowburn, Adv. Funct. Mater. 26, 4704 (2016).
[11] T. Vemulkar, R. Mansell, D. Petit, R. P. Cowburn, and M. Lesniak, Appl. Phys. Lett. 107, 012403 (2015).

[12] Y. Yafet, Phys. Rev. B 36, 3948 (1987).

[13] P. Bruno and C. Chappert, Phys. Rev. Lett. 67, 1602 (1991).

[14] S. S. P. Parkin, N. More, and K. P. Roche, Phys. Rev. Lett. 64, 2304 (1990).

[15] D. Mosca, F. Petroff, A. Fert, P. Schroeder, W. Pratt, and R. Laloee, J. Magn. Magn. Mater. 94, L1 (1991).

[16] W. R. Bennett, W. Schwarzacher, and W. F. Egelhoff, Phys. Rev. Lett. 65, 3169 (1990).

[17] X. M. Liu, H. T. Nguyen, J. Ding, M. G. Cottam, and A. O. Adeyeye, Phys. Rev. B 90, 064428 (2014).

[18] Z. Zhang, L. Zhou, P. E. Wigen, and K. Ounadjela, Phys. Rev. Lett. 73, 336 (1994).

[19] M. Vohl, J. Barnaś, and P. Grünberg, Phys. Rev. B 39, 12003 (1989).

[20] P. Grünberg, J. Appl. Phys. 52, 6824 (1981).

[21] R. Damon and J. Eshbach, J. Phys. Chem. Solids 19, 308 (1961).

[22] R. A. Gallardo, T. Schneider, A. K. Chaurasiya, A. Oelschlägel, S. S. P. K. Arekapudi, A. Roldán-Molina, R. Hübner, K. Lenz, 
A. Barman, J. Fassbender, J. Lindner, O. Hellwig, and P. Landeros, Phys. Rev. Appl. 12, 034012 (2019).

[23] K. Di, S. X. Feng, S. N. Piramanayagam, V. L. Zhang, H. S. Lim, S. C. Ng, and M. H. Kuok, Sci. Rep. 5, 10153 (2015).

[24] R. Camley, Surf. Sci. Rep. 7, 103 (1987).

[25] M. S. Kang, A. Butsch, and P. S. J. Russell, Nat. Photonics 5, 549 (2011).

[26] Z. Wang and S. Fan, Opt. Lett. 30, 1989 (2005).

[27] Z. Zhang, L. Zhou, P. E. Wigen, and K. Ounadjela, Phys. Rev. B 50, 6094 (1994).

[28] P. E. Wigen, Z. Zhang, L. Zhou, M. Ye, and J. A. Cowen, J. Appl. Phys. 73, 6338 (1993).

[29] V. P. Denysenkov and A. M. Grishin, Rev. Sci. Instrum. 74, 3400 (2003).

[30] B. Khodadadi, J. B. Mohammadi, J. M. Jones, A. Srivastava, C. Mewes, T. Mewes, and C. Kaiser, Phys. Rev. Appl. 8, 014024 (2017).

[31] J. Hamrle, J. Pištora, B. Hillebrands, B. Lenk, and M. Münzenberg, J. Phys. D: Appl. Phys. 43, 325004 (2010).

[32] Z. Li, R. Skomski, S.-H. Liou, S. Michalski, M. Chipara, and R. D. Kirby, J. Appl. Phys. 109, 07C113 (2011).

[33] G. Wu, S. Chen, Y. Ren, Q. Y. Jin, and Z. Zhang, ACS Appl. Nano Mater. 2, 5140 (2019).

[34] A. Kamimaki, S. Iihama, T. Taniguchi, and S. Mizukami, Appl. Phys. Lett. 115, 132402 (2019).

[35] A. Prabhakar and D. D. Stancil, Spin Waves: Theory and Applications (Springer, US, 2009).
[36] E. Beaurepaire, J.-C. Merle, A. Daunois, and J.-Y. Bigot, Phys. Rev. Lett. 76, 4250 (1996).

[37] B. Koopmans, J. J. M. Ruigrok, F. Dalla Longa, and W. J. M. de Jonge, Phys. Rev. Lett. 95, 267207 (2005).

[38] M. van Kampen, C. Jozsa, J. T. Kohlhepp, P. LeClair, L. Lagae, W. J. M. de Jonge, and B. Koopmans, Phys. Rev. Lett. 88, 227201 (2002).

[39] A. R. Khorsand, M. Savoini, A. Kirilyuk, and T. Rasing, Nat. Mater. 13, 101 (2014).

[40] See Supplemental Material at http://link.aps.org/supplemental/ 10.1103/PhysRevB.101.214434 for the frequency dependence on the applied magnetic field of the different modes with error bars included.

[41] Z. Li, R. Skomski, S. Michalski, L. Yue, and R. D. Kirby, J. Appl. Phys. 107, 09D303 (2010).

[42] See Supplemental Material at http://link.aps.org/supplemental/ 10.1103/PhysRevB.101.214434 for the evolution of the transient mode on varying the pump laser fluence for sample 2 at $\mu_{0} H=130 \mathrm{mT}$.

[43] See Supplemental Material at http://link.aps.org/supplemental/ 10.1103/PhysRevB.101.214434 for the time constant and the corresponding damping extracted from the fitting for samples 2 and 3.

[44] J. Zhou and S. Saha, dataset for "Ultrafast laser induced precessional dynamics in antiferromagnetically coupled ferromagnetic thin films", doi: 10.5281/zenodo.3837280 (2020). 